\title{
MENSURAÇÃO DO COMPORTAMENTO ESTRATÉGICO A PARTIR DA CULTURA ORGANIZACIONAL: UM ESTUDO EM LOJAS DE SHOPPING CENTERS
}

\section{RESUMO}

Esta pesquisa apresenta como objetivo geral mensurar o comportamento estratégico a partir dos estilos de cultura organizacional, à luz dos modelos de Paz e Mendes (2001) e Miles e Snow (2003). Quanto aos procedimentos metodológicos, o presente estudo é classificado como do tipo explicativo, de natureza quantitativa, conduzido por meio de uma survey analítica. A amostra utilizada foi do tipo probabilística, aleatória simples, composta por 286 gestores lojistas de 6 shoppings centers da cidade de Natal. Através de uma análise de regressão múltipla, foram identificadas nas organizações estudadas o predomínio do estilo cultural empreendedor. Em relação ao comportamento estratégico, identificou-se um perfil transitando entre o estilo analítico e prospector. Estes resultados indicaram uma forte influência do estilo cultural na definição do posicionamento estratégico das organizações. De forma conclusiva, a pesquisa confirma as suposições da teoria quando apontam para uma necessidade de se promover um alinhamento do posicionamento estratégico da organização com a sua cultura organizacional.

Palavras-chave: Comportamento Estratégico; Cultura Organizacional; Shopping Centers.

\section{STRATEGIC BEHAVIOR MEASUREMENT FROM THE ORGANIZATIONAL CULTURE: A STUDY IN SHOPPING CENTERS}

\begin{abstract}
This research presents as general purpose measuring strategic behavior from organizational culture styles, in the light of Peace and Mendes (2001) and Miles and Snow (2003). Regarding the methodological procedures, the present study is classified as type quantitative nature, explanatory conducted through a survey analytics. The sample used was probabilistic, simple random, composed of 286 tenants managers of 6 shopping centers in the city of Natal. Through multiple regression analysis, were identified in the organizations studied the prevalence of cultural style entrepreneur. In relation to strategic behavior, identified a profile transiting between the analytic style and prospector. These results indicated a strong influence of the cultural style in the definition of the strategic positioning of organizations. Conclusively, the research confirms the assumptions of the theory when you point to a need to promote a strategic positioning alignment of organization with your organizational culture.
\end{abstract}

Keywords: Strategic Behavior; Organizational Culture; Shopping Centers. 


\section{MEDICIÓN DE COMPORTAMIENTO ESTRATÉGICO CULTURA ORGANIZACIONAL: UN ESTUDIO EN COMPRAS DE ATRACCIONES}

\section{RESUMEN}

Esta investigación presenta el comportamiento estratégico medida objetivo general de los estilos de la cultura de la organización a la luz de los modelos de la Paz y Mendes (2001) y Miles y Snow (2003). En cuanto a los procedimientos metodológicos, este estudio se clasifica como el tipo de motivos, cuantitativa, realizada a través de un estudio analítico. La muestra utilizada fue probabilístico, aleatorio simple, que consta de 286 inquilinos gestores de 6 centros comerciales en la ciudad de Natal. A través de un análisis de regresión múltiple, que se identificó en las organizaciones estudiaron la prevalencia de estilo empresarial cultural. En cuanto al comportamiento estratégico, hemos identificado un perfil en tránsito entre el estilo analítico y buscador de oro. Estos resultados indican una fuerte influencia del estilo cultural en la definición del posicionamiento estratégico de las organizaciones. En conclusión, la investigación confirma los supuestos de la teoría cuando apuntan a la necesidad de promover una alineación de posicionamiento estratégico de la organización con su cultura organizacional.

Palabras clave: Comportamiento Estratégico; Cultura de la Organización; Centros Comerciales.

Miler Franco Danjour ${ }^{1}$ Bruno Campelo Medeiros ${ }^{2}$ Miguel Eduardo Moreno Añez ${ }^{3}$ Afrânio Galdino de Araújo ${ }^{4}$

\footnotetext{
${ }^{1}$ Doutorando em Administração pela Universidade Federal do Rio Grande do Norte - UFRN. Brasil. E-mail: miler.danjour@ifrn.edu.br

${ }^{2}$ Doutorando em Administração pela Universidade Federal do Rio Grande do Norte - UFRN. Brasil. E-mail: bruno.campelo@ifrn.edu.br

${ }^{3}$ Doutor em Administração de Empresas pela Escola de Administração de Empresas de São Paulo da Fundação Getulio Vargas - EAESP/FGV.Professor da Universidade Federal do Rio Grande do Norte - UFRN. Brasil. E-mail: anez1957@yahoo.com.br

${ }^{4}$ Doutor em Engenharia de Produção pela Universidade Federal de Pernambuco - UFPE. Professor da Universidade Federal do Rio Grande do Norte - UFRN. Brasil. E-mail: afranioga@gmail.com
} 


\section{INTRODUÇÃO}

A cultura organizacional passa a ser estudada e aplicada ao mundo dos negócios com o surgimento da abordagem comportamental das organizações que se propõem a estudar as manifestações sociais do indivíduo e sua forma de interferência em um contexto organizacional. Ela move a organização para uma direção, sendo esta direção correta ou não, apresentando raízes profundas que, de certa forma, representam o DNA da organização (Carvalho \& Ronchi, 2005; Dutra, Fleury, \& Ruas, 2006; Almeida, Irber, \& Souza, 2013).

Por conseguinte, é importante observar que, sendo a cultura organizacional responsável por influenciar a formação das crenças e valores de uma empresa, ela influencia também o comportamento estratégico. Uma estratégia que provavelmente esteja em desacordo com a cultura da organização dificilmente será implementada, e quando a for, não alcançará sucesso. A cultura pode gerar resistências ou favorecer o processo estratégico das organizações. Portanto, as mudanças estratégicas precisam ser baseadas em mudanças fundamentais na cultura (Carvalho \& Ronchi, 2005; Mintzberg, Ahlstrand, \& Lampel, 2000; Heinzmann \& Machado, 2015).

Schein (2009) reconhece a importância da cultura corporativa na gestão organizacional. Este autor destaca a influência dos elementos culturais na maneira como a empresa opera, nas formas como as pessoas se comportam e no estabelecimento das estratégias organizacionais. Assim, para entender as novas demandas ambientais, as organizações vêm agregando às suas práticas administrativas ferramentas de gestão capazes de compreender o processo complexo da mudança organizacional, bem como suas implicações para a ampliação de mercado e ganho de competitividade.

É válido atentar para as mudanças ocorridas na configuração do meio urbano brasileiro, no qual vem sofrendo alterações consideráveis, recebendo maior destaque as mudanças resultantes das manifestações relacionadas ao setor terciário, como os serviços especializados e os shopping centers. O shopping center deve ser entendido como um "centro de compras" ou "centro comercial", onde existe um grupo de negócios de varejo constituído de forma planejada que é gerido por uma unidade (Kotler \& Armstrong, 1994). Essa nova forma de consumo passou a competir não só com o tradicional comércio varejista de rua e com varejos não lojistas, mas também entre si. Sendo assim, cada empresa se utiliza de estratégias com intuito de atingir a maior parcela de compradores.

No Brasil, o surgimento e a expansão dos shopping centers se deram atrelados às importantes transformações decorrentes da economia brasileira, a partir da implementação do que Gaeta (1992) denominou de "racionalidade burguesa" (décadas de 1950 e 1960), em outras palavras, isso ocorreu quando se fomentava a acumulação do capital no país. Surge, então, uma nova dinâmica de mercado consumidor, que se reflete no desenvolvimento no cenário nacional.

Neste contexto, observa-se o processo de evolução do mercado de shopping centers no Brasil entre os anos de 2000 e 2013, no qual passou de 281 para 382 unidades, o que representa um total de $26,44 \%$ de aumento, gerando, assim, um aumento de $56 \%$ no faturamento desse segmento. Ao observar os números do mercado de shopping centers na cidade de Natal/RN, observa-se um total de 6 shoppings distribuídos entre as Zonas Norte, Sul e Leste da cidade. Esses shoppings possuem um total de 748 lojas, sendo o Midway Mall o maior, com 270 lojas e o Norte Shopping o menor com um total de 70 lojas (Abrasce, 2014).

Diante deste contexto, o presente trabalho coloca o seguinte problema de pesquisa: em que medida os estilos de cultura organizacional influenciam o comportamento estratégico em lojas de shopping centers? Assim, têm-se como objetivo geral da pesquisa mensurar o comportamento estratégico a partir dos estilos de cultura organizacional presentes em lojas dos shopping centers da cidade de Natal, à luz dos modelos de Paz e Mendes (2001) e Miles e Snow (2003). Observando a importância dada à cultura organizacional em relação ao processo de formulação de estratégias, este estudo nasce da necessidade de se iniciar uma discussão sobre os novos parâmetros e diretrizes estratégicas na gestão das micro e pequenas empresas que atuam em shopping centers na cidade do Natal/RN, ao identificar empiricamente uma possível relação entre cultura organizacional com as tipologias de estratégias genéricas utilizadas por essas organizações, de forma que se possa contribuir e estimular formulações de novos estudos, atentando para a aplicação dos conceitos de estratégia na gestão destes empreendimentos, e levando em consideração alguns estudos anteriores que apresentam esta relação, utilizando outras estruturas teóricas (Tomassini, 2001; Pereira e Nunes, 2002; Heinzmann \& Machado, 2015), ou os mesmos modelos utilizados como base para a realização deste trabalho, porém, considerando outras realidades em suas análises (Degenhardt, 2006).

Para responder ao problema de pesquisa, foram utilizados como procedimentos metodológicos uma pesquisa do tipo survey, utilizando-se de uma amostra probabilística e aleatória simples, com técnicas de análise descritiva e de regressão múltipla. Em se tratando de implicações práticas, a pesquisa proporcionou às empresas pesquisadas uma clareza nas relações entre o estilo cultural organizacional e o comportamento estratégico vigente, apontando para uma necessidade de compreensão da cultura como aspecto condicionante para a definição de uma estratégia adequada ao posicionamento e interesse das empresas em geral.

Para as empresas, esta pesquisa proporcionou uma maior compreensão em relação ao processo de 
mudanças vivenciadas pelo ambiente organizacional, no qual estas organizações estão inseridas, uma vez que ao se estudar as relações causais existentes entre a cultura organizacional e a tipologia estratégica adotada por estas organizações, este estudo poderá proporcionar aos gestores um maior entendimento em relação ao comportamento da organização frente às mudanças vivenciadas, além de ampliar os subsídios para o processo decisório destas instituições.

Sendo assim, o presente artigo está dividido nas seguintes partes: Cultura Organizacional; Estratégia Organizacional; Cultura x Estratégia; Procedimentos Metodológicos; Análise dos resultados; Discussão; e Considerações Finais.

\section{CULTURA ORGANIZACIONAL}

Diante das transformações no mundo empresarial, ocorre no início do século XX a inserção da ideia de cultura organizacional nas corporações norte-americanas. Entretanto, apenas nos anos de 1980, passou-se a dar determinada atenção a esse tema fundamental para as organizações. Com o surgimento da abordagem comportamental organizacional, na qual é proposto o estudo das manifestações sociais dos indivíduos, passou-se de fato aplicar a cultura nas organizações.

A maneira de ver as organizações como culturas é um fato relativamente recente, que para Robbins (2005), Almeida et al. (2013), até meados da década de 1980, quase sempre, as organizações eram visualizadas apenas de uma forma lógica de coordenar e inspecionar um grupo de pessoas. No entanto, as organizações são mais complexas que isso. Elas possuem individualidades, assim como os indivíduos. Quando uma organização se torna instituição, ela adquire uma independência de seus patriarcas ou de qualquer um de seus membros.

Segundo Zaccarelli (1986), o conceito de cultura organizacional surge dentro de uma perspectiva funcionalista, de forma que se destaca a visão de Schein (2009, p. 16) que conceitua cultura organizacional como um padrão de suposições básicas compartilhadas, aprendido por um grupo à medida que solucionava seus problemas de adaptação e integração. Esse padrão tem funcionado bem o suficiente para ser considerado válido e, por conseguinte, para ser ensinado aos novos membros como modo correto de perceber, pensar e sentir-se em relação a esses problemas.

Tendo como premissa a visão de Schein, aliada a elementos políticos, torna-se viável a concepção de Fleury e Fischer (2007) a respeito da cultura organizacional, como sendo um conjunto de valores e pressupostos básicos expressos em elementos simbólicos, que tem sua capacidade de ordenar, atribuir significações, e construir uma identidade organizacional.

Segundo Robbins (2005), Monteiro, Palma e Lopes (2012), uma consistente cultura organizacional pode nortear a empresa, além de direcionar os funcionários, como também o fato de institucionalizar equilíbrio e segurança para ela. Contudo, algumas empresas e organizações vêem a cultura com "maus olhos", pois uma cultura forte e consistente pode ser um fator negativo para a realização de mudanças. Deste modo, Motta e Caldas (1997) ressalta para a força que a cultura organizacional pode apresentar, tanto em efeitos positivos, quanto negativos para a organização e seus indivíduos.

\subsection{Cultura organizacional a partir do modelo de Paz e Mendes}

Segundo Paz e Mendes (2001), cada vez mais cresce a vontade do homem de conhecer o seu mundo, no qual estão inseridos grupos sociais nas organizações de trabalho, sendo imprescindível a compreensão acerca das organizações. Nesse contexto, foi construída a Escala de Estilos de Funcionamento Organizacional (EEFO), para que se pudessem avaliar certas formas de sentir, pensar e agir compartilhadas nas organizações, a fim de compreendê-las em uma perspectiva cultural. Dentro desta visão, alguns autores se propuseram a desenvolver modelos e visões para a análise da cultura organizacional, baseados em diversos aspectos, sejam relacionados à análise da cultura dominante e das subculturas (Robbins, 2005), aos níveis de cultura (Pettigrew, 2007; Cameron \& Quinn, 2006, Schein, 2009), ou à inovação e sistema dinâmico (Zago, 2013).

A partir da visão clássica, percebe-se a ligação do conceito de cultura com o processo de desenvolvimento organizacional, sendo ela um instrumento de melhorias a partir da concepção de valores. Algumas décadas depois, os autores disseminaram a importância de se estudar a cultura organizacional para conduzir a empresa ao sucesso. A convivência em um novo mundo de avanços tecnológicos e informacionais acaba influenciando no processo de tomada de decisões e maneiras de comportamentos dentro das organizações, fatores que refletem diretamente na cultura por meio de novas práticas.

É válido lembrar que a implantação de um modelo inovador será mais viável a organizações que já possuem um estilo de cultura mais apto à aceitação de mudanças. Isso pode ser observado na Figura 1, onde é apresentada a Escala de Estilos de Funcionamento Organizacional de Paz e Mendes (2008), composta por cinco estilos (Individualista, Burocrático, Afiliativo, Empreendedor e Cívico) objetivando a pesquisa do funcionamento organizacional no que tange a relação entre a organização e os agentes organizacionais. 
Mensuração do Comportamento Estratégico a Partir da Cultura Organizacional: Um Estudo em Lojas de Shopping Centers

Figura 1 - Escala de Estilos de Funcionamento das Organizações (EEFO)

\begin{tabular}{|c|c|}
\hline $\begin{array}{c}\text { ESTILO DE } \\
\text { FUNCIONAMENTO }\end{array}$ & MODO DE PENSAR, SENTIR E AGIR \\
\hline INDIVIDUALISTA & $\begin{array}{l}\text { Apresenta modos de pensar, sentir e proceder, compartilhados pelos membros que } \\
\text { refletem em uma fusão indivíduo e organização, de forma que o predomínio desse } \\
\text { estilo levará a atitudes direcionadas ao próprio ego. }\end{array}$ \\
\hline BUROCRÁTICO & $\begin{array}{l}\text { Apresenta formas de pensar, sentir e agir que se baseiam no controle das normas e } \\
\text { regras, de maneira que os indivíduos pertencentes a esse tipo de cultura apresentam um } \\
\text { envolvimento parcial com organização em prol da segurança, oferecendo resistência as } \\
\text { mudanças, as quais são vistas como ameaças pelos integrantes. }\end{array}$ \\
\hline AFILIATIVO & $\begin{array}{l}\text { Revela uma forma de pensar, sentir e agir que apresenta a organização como um grupo } \\
\text { impecável, no qual é refletido o orgulho, a confiança e a autoestima da organização. } \\
\text { Nessa cultura, a organização é vista pelos seus integrantes como algo perfeito, na qual } \\
\text { as imperfeições são de origem externa. Assim, o comportamento dos membros desse } \\
\text { tipo de cultura é de evitar os conflitos e colocam os interesses da organização acima de } \\
\text { tudo. }\end{array}$ \\
\hline EMPREENDEDOR & $\begin{array}{l}\text { Caracteriza-se por revelar formas de pensar, sentir e proceder voltados para os } \\
\text { empreendimentos, na qual os seus integrantes estão sempre buscando novos desafios. } \\
\text { O comportamento desses integrantes está voltado para necessidade de reconhecimento } \\
\text { pela sua atuação na organização, sendo sempre ativos e vendo as frustrações como } \\
\text { obstáculos a serem superados. }\end{array}$ \\
\hline CÍVICO & $\begin{array}{l}\text { Desvela um jeito de pensar, sentir e agir voltados às relações de reciprocidade. Nesse } \\
\text { tipo de cultura a organização investe na admiração dos seus membros de acordo com a } \\
\text { atuação deles. Neste estilo, as regras estão abertas a críticas e modificações. Além } \\
\text { disso, valoriza princípios e ideias de bens comum, fazendo com que seus membros } \\
\text { estejam sempre buscando o crescimento, obedeçam às regras e apreciem o trabalho } \\
\text { exercido. }\end{array}$ \\
\hline
\end{tabular}

Fonte: Adaptado de Paz e Mendes (2008).

Assim, o modelo apresentado por Paz e Mendes (2008) vislumbra uma compreensão das diferentes formas de manifestação da cultura, a partir dos padrões comportamentais compartilhados pela maioria dos membros da organização, de forma que, a maneira como essa se apresenta impactará cada organização de forma negativa ou positiva, visto que, alguns estilos apresentam aspectos mais voltados para o individualismo, enquanto outros apresentam características voltadas para o bem comum. Dependendo do estilo adotado, a organização se caracterizará de forma mais flexível ou mais resistente a mudanças que possam ser necessárias para seu avanço.

\section{ESTRATÉGIA ORGANIZACIONAL}

Em termos de estratégia, há uma grande diversidade de conceitos. $\mathrm{O}$ ecletismo sobre as definições da estratégia faz com que ocorra uma complementação entre esses conceitos. Cada definição tem o seu mérito e a sua devida importância histórica, sendo possível observar que o termo estratégia norteou todo o processo de evolução da humanidade. Como afirma Hambrick (1980), Pedrozo e Rodriguez (2013), a estratégia pode ser apresentada sob uma ótica multidimensional e situacional.

As organizações nascem com propósitos, às vezes não tão claros, da vontade de seus fundadores, que nem sempre estão envolvidos com o mercado (Lourenço \& Ferreira, 2012; Powell, 2014). É necessário introduzir o processo estratégico a fim de adotar medidas cruciais para o futuro da organização. Assim, Mintzberg et al. (2006) define processo estratégico como sendo um processo de moldagem no qual as estratégias efetivas ganham vida, e onde a formulação e a implementação se unem em um processo contínuo de aprendizado pelo qual as estratégias criativas se desenvolvem, em meio a um contexto complexo ambiental, na qual a estratégia é moldada pelos recursos organizacionais, e, ao mesmo tempo, sofre influência das variáveis externas. 


\subsection{O comportamento estratégico a partir do modelo de Miles e Snow}

Identificar os tipos de comportamento estratégico, os quais variam a partir da dinamicidade do processo de adaptação ao ambiente organizacional, é importante para a tomada de decisões e alinhamento estratégico para manter a competitividade. Por conseguinte, desenvolveu-se uma diversidade de modelos e visões acerca do tema, os quais contribuem para a sua compreensão.

Dentre esses modelos, destaca-se o modelo teórico de Miles e Snow (2003). Para estes autores, as empresas desenvolvem padrões de comportamento estratégico relativamente estáveis para se alcançar o alinhamento com as condições ambientais (Lang et al., 2014). De acordo com os padrões de resposta às condições ambientais, os autores propõem uma tipologia de análise estratégica formada por quatro tipos de estratégias genéricas, conforme demonstrada no Quadro 2.Cada uma das estratégias tem características distintas para responder à diversidade ambiental, como a configuração de tecnologia, estrutura e processo que são consistentes com essas estratégias (Miles \& Snow, 2003; Zanin, Machado, \& Sehnem, 2011; Martins et al., 2014; Powell, 2014). A relação entre os quatro tipos de estratégia e o ambiente é o conceito central do modelo proposto. Tal tipologia se reduz a duas formas básicas (as quais parecem corresponder às organizações máquinas e adhocracia), sendo desdobradas em outras duas, onde uma consiste em um formato híbrido e outra como uma coleção de respostas inadequadas (Mintzberg et al., 2000). Essa inconsistência é apontada anteriormente pelos autores da estratégia reativa adotada por algumas organizações. Miles e Snow (2003) apontam três causas básicas:

- A primeira está relacionada à falha da administração na articulação de uma estratégia viável;

- A segunda sugere que a estratégia é articulada, mas a tecnologia, a estrutura, e os processos não estão vinculados a ela de maneira adequada;

- E por fim os autores apontam para vinculação da administração a uma estratégia e estrutura que não são adequadas as condições ambientais.

Deste modo, a Figura 2 explica as principais características das tipologias resultantes do estudo desenvolvido por Miles e Snow (2003) em quatro setores industriais. Os autores apresentam o modelo de tipologias de estratégias genéricas, que por ser abrangente podem ser aplicadas em empresas de qualquer porte, sendo proposto que, dependendo da categoria de estratégia adotada, a empresa apresentará um diferencial competitivo específico.

Figura 2 - Tipologias das estratégias de Miles e Snow

\begin{tabular}{|c|c|}
\hline ESTRATÉGIA & CARACTERÍSTICAS \\
\hline PROSPECTIVA & $\begin{array}{l}\text { - Designada para aprendizagem; estrutura adaptável e descentralizada. } \\
\text { - Forte aptidão em pesquisa. }\end{array}$ \\
\hline DEFENSIVA & $\begin{array}{l}\text { - Orientação para eficiência; comando centralizado e rigidez no controle de custos. } \\
\text { - Atenção voltada para eficiência da produção; baixas despesas administrativas. } \\
\text { - Supervisão rigorosa: pouco empowerment dos funcionários. }\end{array}$ \\
\hline ANALISADORA & $\begin{array}{l}\text { - Equilíbrio entre eficiência e aprendizagem; supervisão cerrada de custos com } \\
\text { flexibilidade e adaptabilidade. } \\
\text { - Eficiência na produção da linha de produtos estáveis; realce na importância da } \\
\text { pesquisa, criatividade, tomada de risco para inovação. }\end{array}$ \\
\hline REATIVA & $\begin{array}{l}\text { - Nenhuma abordagem clara no âmbito da organização; alta volatilidade das } \\
\text { características do projeto, dependendo de suas necessidades presentes. }\end{array}$ \\
\hline
\end{tabular}

Fonte: Adaptado de Miles e Snow (2002).

A primeira é a estratégia Prospectora. Conforme Martins et al. (2008; 2014) essas organizações apresentam uma postura competitiva e agressiva, devido à vontade de estar sempre à procura de novas oportunidades. Por realizar altos investimentos em pesquisa e em novos produtos, acabam por correrem altos riscos em caso de uma possível não aceitação do novo produto ou serviço. Miles e Snow (2003) afirmam que devido a permanente procura por inovações, há uma tendência de perda parcial da eficácia.
A estratégia Defensiva envolve organizações que possuem pouco conhecimento acerca de produtomercado. Assim, elas possuem barreiras para possíveis mudanças na parte tecnológica, estrutural ou método de operações, admitindo o foco na exatidão das operações. Seguindo esse mesmo raciocínio, Scherer e Mussi (2000) atestam que essas empresas buscam a criação de um conjunto consistente de produtos e/ou serviços que são conduzidos a um segmento de mercado específico. 
A estratégia Analítica apresenta características intermediárias entre as estratégias defensiva e prospectora, de acordo com Martins et al. (2008). Para Miles e Snow (2003), as organizações que trabalham com esse tipo de estratégia acabam funcionando com dois tipos de produto-mercado, um bastante rígido, no qual não se modifica, e outro que é suscetível à mudança. Para Bott et al. (2006), o produto-mercado rígido se utiliza de estrutura e mecanismos padronizados e axiomatizados, enquanto que no produto-mercado em mudança as estratégias mais auspiciosas da concorrência são utilizadas pelas organizações analíticas.

A última estratégia é a Reativa. Para Miles e Snow (2003) os gestores conseguem ter uma visão perceptível referente às mudanças e as dúvidas presentes na organização, no entanto, não conseguem tomar decisões capazes de mudar essa realidade de forma eficaz, só reagindo às pressões de outras empresas por meio de pressões. Devido a isso, se encontram em desvantagem por não conseguirem ter uma postura proativa capaz de equipará-la as outras organizações. Este modelo, portanto, se caracteriza pela falta de alinhamento entre ações, processos, e estrutura (Vidal, Barbosa, \& Bouzada, 2014).

Assim, pode-se perceber que cada tipo de estratégia do modelo proposto carece de um ajuste particular de tecnologia, estrutura e outros processos envolvidos, não havendo a predominância de um tipo sobre outro, pois fica visível o estado de interdependência entre as diversas áreas funcionais da empresa.

\section{CULTURA X ESTRATÉGIA: UMA RELAÇÃO ORGANIZACIONAL}

Cada organização possui uma cultura própria que é repassada aos colaboradores que se adaptam e acabam compreendendo melhor o funcionamento como um todo da organização. A cultura organizacional é a identidade da empresa, sendo essa variável formada por um conjunto de características típicas como normas, crenças e valores (Powell, 2014). Para Srour (1998), nas organizações, a cultura impregna todas as práticas, sendo um elemento que influencia todos os outros elementos.

Porter (1986) ressalta a importância de adicionar o conhecimento a respeito das crenças e de outras características culturais às ferramentas gerenciais aplicadas as estratégias de negócios. Ele afirma ainda ser fortuito acrescentar que o saber acerca da cultura da organização é desejável para o estabelecimento de condições organizacionais apropriadas para administrar as mudanças decorrentes de estratégias, seus impactos e ressonâncias.

A estratégia e a cultura organizacional possuem forte relação de interdependência. Trata-se de uma relação íntima, observada principalmente no momento de se avaliar os rumos que a organização deve tomar.
Colaborando com essa percepção, Daft (2002) afirma que a cultura deve reforçar a estratégia e o projeto estrutural da organização, pois o sucesso ou fracasso desta, na maioria das vezes, é atrelado a sua cultura. O autor conclui o seu pensamento dizendo que a boa ou má gestão da cultura poderá influenciar a sobrevivência da organização.

A relação entre os conceitos de cultura e estratégia ocorre em muitas e variadas ligações. Sendo assim, Mintzberg et al. (2000) resumiram algumas dessas ligações, de acordo com o desenvolvimento da literatura:

- Estilo de tomada de decisão - Cada organização possui sua cultura específica, e ela por sua vez influencia a maneira de pensar, analisar e agir de uma organização como um todo. Assim, a cultura acaba influenciando o processo de formação de estratégia. Neste estilo, em outras palavras, a cultura funciona como agente filtrante para as tomadas de decisões.

- Resistência a mudanças de estratégias - As organizações que possuem crenças bem enraizadas acabam passando por um desencorajamento nas mudanças de suas estratégias.

- Superar a resistência às mudanças estratégicas - Este ponto desperta para o risco da estagnação decorrente de uma cultura resistente. Deve-se adotar a visão do novo, da flexibilidade. Um sistema fechado tende a caminhar para o fracasso. Mintzberg et al. (2000) sugerem diversas formas de lutar contra essa realidade, inclusive com a nomeação de um alto executivo sem cargo, para fazer questionamentos e dar ideias, por exemplo.

- Valores dominantes - Nesse quesito, fica em evidência a importância de valores-chave para o sucesso das empresas. Valores como a qualidade, o atendimento e a inovação promovem vantagens competitivas, e estas, por sua vez, servem para sustentar perspectivas estratégicas notavelmente estáveis.

- Choque de Culturas - Os autores apresentam esse fator como um motivo de conflito entre as empresas que tentam fundir-se. A cultura organizacional é a identidade da empresa. Dessa forma, é provável que as diferentes leituras sobre o ambiente e os acontecimentos, gerem uma zona de divergência, muitas vezes impossível de ser solucionada.

Com o passar do tempo, ocorreu uma evolução acerca das visões dos autores e estes passaram, cada vez mais, a formular conceitos a partir de aspectos que mostrassem a relação entre as culturas e as estratégias 
organizacionais. No entanto, mesmo com uma significativa quantidade de trabalhos que abarcam esses temas isoladamente, existe uma reduzida quantidade de trabalhos que apresentam estudos direcionados às relações entre estes temas. Com isso, a Figura 3 a seguir mostra alguns desses exemplos.

Figura 3 - Visões e aspectos da relação cultura organizacional e estratégia organizacional

\begin{tabular}{|c|c|}
\hline AUTORES & ASPECTOS DESTACADOS \\
\hline Johnson (1992) & $\begin{array}{l}\text { As dimensões sociais, políticas, culturais e cognitivas das atividades } \\
\text { gerenciais, dão origem as mudanças estratégicas tanto incrementais } \\
\text { quanto fundamentais para a organização. }\end{array}$ \\
\hline $\begin{array}{l}\text { Tomassini (2001), Pereira e Nunes } \\
\text { (2002), Degenhardt (2006), Sampaio, } \\
\text { Freitas e Máximo (2007) }\end{array}$ & $\begin{array}{l}\text { A influência da cultura na estratégia organizacional e o } \\
\text { relacionamento entre elas. }\end{array}$ \\
\hline Afonsêca, Teodósio e Paixão (2011) & $\begin{array}{l}\text { Teoria Institucional; } \\
\text { Cultura corporativa; } \\
\text { Análise de papéis sociais de Goffman. }\end{array}$ \\
\hline Ravishankar, Pan e Leidner (2011) & $\begin{array}{l}\text { Estabelece relações entre o desenvolvimento da estratégia } \\
\text { organizacional, com as dimensões da cultura e a ação gerencial }\end{array}$ \\
\hline Lourenço e Ferreira (2012) & $\begin{array}{l}\text { A influência do fundador; } \\
\text { Mudanças após sua morte; } \\
\text { Associação da imagem do fundador a de um herói. }\end{array}$ \\
\hline Castro e Silva (2012) & $\begin{array}{l}\text { A orientação estratégica exerce influencia sobre a cultura, todavia, } \\
\text { essa relação não apresenta entre esse não construtos, não } \\
\text { modificaram o modelo de gestão de pessoas da organização. }\end{array}$ \\
\hline $\begin{array}{l}\text { Monteiro, Palma e Lopes (2012), } \\
\text { Almeida et al. (2013) }\end{array}$ & $\begin{array}{l}\text { Capital empreendedor; } \\
\text { Cultura Inovadora; } \\
\text { Culturas influenciadas pela orientação externa. }\end{array}$ \\
\hline $\begin{array}{l}\text { Pedrozo e Rodriguez (2013); Ferreira, } \\
\text { Bubach e Silva Júnior (2014) }\end{array}$ & $\begin{array}{l}\text { Complexidade e pluralismo. Influência de agentes externos; } \\
\text { Estrategista inteirado com o processo dos feedbacks. }\end{array}$ \\
\hline Heinzmann e Machado (2015) & $\begin{array}{l}\text { Valores e dimensões; } \\
\text { Estágios de internacionalização. }\end{array}$ \\
\hline
\end{tabular}

Fonte: Dados da pesquisa, 2016.

O modo como uma organização deve se constituir para perseguir uma estratégia depende de um complexo conjunto de fatores, estrutura, sistemas, pessoas e cultura, visto que não se pode alcançar um desempenho favorável em um ambiente onde não há sinergia entre essas dimensões. Em outras palavras, afirmar que a eficiência e a eficácia organizacional dependem do ajuste entre a cultura organizacional e a orientação estratégica pressupõe a criação de um sistema adequado, a fim de reforçar os propósitos definidos pelas organizações (Klein, 2008).

A partir da discussão desencadeada pelos teóricos presentes no quadro, nota-se a importância de se inteirar do modelo de cultura antes de implementar a estratégia, é necessário analisar a cultura vigente para identificar as crenças e valores da organização. A cultura empresarial precisa ser bem determinada, para que ela possa orientar as estratégias desenvolvidas, uma vez que as etapas do processo de planejamento estratégico estão relacionadas com os aspectos da cultura organizacional.

De acordo com a contextualização apresentada, foram escolhidos os modelos Paz e Mendes (2008) para definir os estilos de cultura organizacional e Miles e Snow (2003) para definir o comportamento estratégico. Em relação à escolha do modelo de cultura organizacional, foram levadas em consideração a adequação do modelo à realidade nacional das organizações e suas características, não necessitando a realização de uma redução sociológica das variáveis do instrumento para compreender a realidade do campo de pesquisa.

Com relação à escolha do modelo de Miles e Snow (2003) para estudar o comportamento estratégico organizacional, partiu-se da adequação do modelo à realidade de empresas que atuam e diversos setores da economia, uma vez que os modelos que estudam estratégias genéricas, como Porter (1986) são mais adequados para a realidade da indústria.

Diante do levantamento teórico realizado, a presente pesquisa levanta a seguinte hipótese: o estilo de cultura organizacional influencia no comportamento estratégico de lojas de shopping centers. 


\section{PROCEDIMENTOS METODOLÓGICOS}

Quanto aos objetivos, o presente estudo classifica-se como sendo do tipo explicativo. Segundo Collis e Hussey (2005, p. 71) esse tipo de pesquisa "tem como objetivo entender os fenômenos, descobrindo e mensurando relações causais entre eles". Quanto aos procedimentos, este estudo será classificado como sendo uma survey analítica, que segundo Collis e Hussey (2005, p. 71) é uma metodologia positivista que a partir de uma amostra representativa da população permite realizar inferências sobre esta, além de "determinar se há alguma relação entre diferentes variáveis", necessitando que o pesquisador identifique as variáveis dependentes e independentes do seu estudo.

A utilização desse procedimento de coleta se deve, sobretudo, a dois fatores. Em primeiro lugar, porque os estudos que tratam de cultura organizacional como causa influenciadora na definição do comportamento estratégico têm utilizado de forma predominante métodos e técnicas de aprofundamento contextual dessa relação, tratando com objetos de estudo casos específicos de realidades distintas. Há portanto, a necessidade de utilizar procedimentos que demonstrem de uma forma mais generalista este fenômeno de investigação. Segundo, porque esta pesquisa segue uma das recomendações propostas por Creswell (2010), considerando que o levantamento se traduz em um processo mais rápido de coleta de dados, considerando o volume de empresas na qual se pretendeu investigar. Por fim, quanto à natureza, a pesquisa é quantitativa no que diz respeito à classificação das estratégias genéricas, tipologia de cultura organizacional e estabelecimento das possíveis relações entre estas variáveis.

Em relação ao instrumento de pesquisa, este foi construído através de uma escala variando de 1 a 5 pontos, tanto para cultura quanto para estratégia. Este instrumento foi elaborado com base no modelo teórico proposto por Paz e Mendes (2008) - denominado de Escala de Estilos de Funcionamento Organizacional EEFO para identificar a presença de duas tipologias culturais nas instituições estudadas. Para isso, foi aplicado um questionário composto por 11 variáveis para cultura, onde os valores próximos de 1 representam um estilo cultural burocrático, e próximo a 5 um estilo cultural empreendedor.

Para identificar as estratégias organizacionais, utilizou-se o modelo teórico de Miles e Snow (2003), adaptado do instrumento desenvolvido por Conant, Monkwa e Varadajan (1990) para avaliar estratégias genéricas existentes nas organizações estudadas. Assim, foi aplicado um questionário composto por 11 variáveis para estratégia no qual os valores próximos de 1 representam uma estratégia defensiva e próximo a 5 uma estratégia prospectiva. Para isto, foram definidas faixas para diagnosticar em cada observação estudada qual era o perfil de cultura organizacional predominante, bem como de comportamento estratégico, de acordo com a Tabela 1 em seguida:

Tabela 1. Escalas de mensuração da cultura organizacional e do perfil estratégico.

\begin{tabular}{|l|l|}
\hline \multicolumn{2}{|c|}{ Cultura Organizacional } \\
\hline \multicolumn{1}{|c|}{ Tipo } & \multicolumn{1}{c|}{ Estilo de Cultura (Grau) } \\
\hline Estilo Empreendedor & $\mathrm{GC} \geq 3,5$ \\
\hline Estilo Afiliativo & $\mathrm{GC}<3,5$ e GI $\geq 2,5$ \\
\hline Estilo Burocrático & $\mathrm{GC}<2,5$ \\
\hline \multicolumn{2}{|c|}{ Estratégia } \\
\hline \multicolumn{1}{|c|}{ Tipo } & \multicolumn{1}{c|}{ Comportamento Estratégico (Grau) } \\
\hline Prospectiva & $\mathrm{GE} \geq 3,5$ \\
\hline Analisadora & $\mathrm{GE}<3,5$ e GI $\geq 2,5$ \\
\hline Defensiva & $\mathrm{GE}<2,5$ \\
\hline
\end{tabular}

Fonte: Dados da pesquisa, 2016.

Além destas variáveis, o instrumento de pesquisa também contemplou questões relativas ao perfil das organizações pesquisadas, como porte da empresa, situação em relação à marca (ser franqueada ou não), tempo de atuação no mercado e número de funcionários. Estas questões foram utilizadas como variáveis de controle, complementando o modelo estruturado neste estudo.
A população deste estudo foi composta por 748 (setecentos e quarenta e oito) gestores das lojas pertencentes aos 6 (seis) shopping centers situados na cidade do Natal/RN. Foi utilizado como procedimento de escolha da amostra, a amostra probabilística aleatória simples, onde, para uma população finita, tem-se o cálculo através da seguinte equação: 
Figura 4 - Equação do cálculo amostral

$$
n=\frac{N Z \alpha^{2} \sigma^{2}}{s^{2}(N-1)+Z \alpha^{2} \sigma^{2}} \text {, em que: }
$$

$\mathrm{n}=$ amostra

$\mathrm{N}=$ população

$Z \alpha=$ escore normalizado para estimativas de confiança estatística

$\varepsilon=$ erro amostral

$\sigma=$ desvio-padrão

Fonte: Adaptado de Fonseca e Martins (1996).

De acordo com o cálculo realizado, para um erro amostral de $5 \%$ e confiabilidade de $95 \%$, o estudo deveria contar com uma amostra de 255 gestores das lojas pertencentes aos 6 (seis) shopping centers estudados. Sendo assim, foram enviados 286 questionários, aplicados presencialmente pelos alunos de iniciação científica e os pesquisadores com os gestores das lojas dos 6 shopping centers da cidade do Natal/RN, no período de maio a julho de 2014, tendo retornado 240 questionários em condições de tabular. Mesmo obtendo uma amostra inferior à recomendada pelo cálculo amostral, para Hair Jr. et al. (2005), esta amostra é relevante, pois, segundo o autor, deve-se ter, pelo menos, 5 respondentes para cada variável utilizada na pesquisa.

Os dados coletados foram analisados objetivando identificar a natureza das relações entre cultura organizacional com o posicionamento estratégico presentes nas organizações estudadas. Para tal, foi utilizada a análise de regressão múltipla. Para Wooldridge (2013, p. 64) a regressão múltipla é um modelo que "permite controlar explicitamente muitos outros fatores que, de maneira simultânea, afetam a variável dependente". Para efeito desse estudo foi considerada como variável dependente as estratégias adotadas pelas organizações. As variáveis independentes foram consideradas como sendo os "estilos de cultura organizacional" (CultOrg), bem como as variáveis de controle "Número de Funcionários" (Funcionários), "Tempo de atuação da Empresa no mercado" (TempoAtuação), além de variáveis dummy representando o porte da empresa, sendo representadas por "Pequena Empresa" (PequenaEmp), "Média Empresa" (MediaEmp), "Média para Grande" (MediaGrandeEmp) e "Grande Empresa" (GrandeEmp). Também foi incluída a variável "Franquia" (dummy), sendo atribuído o valor 1 caso a loja em questão seja uma franquia.

Os parâmetros dos modelos gerados para esta pesquisa foram estimados seguindo o método de mínimos quadrados ordinários (MQO). O método de mínimos quadrados ordinários é usado para estimar os parâmetros de inclinação e de intercepto de um modelo populacional, de modo a minimizar a soma dos quadrados dos resíduos (Wooldridge, 2010; Hair Jr. et al., 2005). No intuito de obter intervalos de confiança mais precisos, foi utilizado no modelo de regressão robusto. Para realizar a análise de regressão, teve-se como base o uso do software Stata (versão 12).

Por fim, buscando assegurar os padrões éticos da pesquisa quantitativa e, com o objetivo de fortalecer a transparência, o instrumento de pesquisa, e o banco de dados poderão ser disponibilizados mediante solicitação aos autores.

\section{ANÁLISE DOS RESULTADOS}

Com o objetivo de se estudar o perfil cultural e estratégico em organizações de shopping centers na cidade de Natal/RN, buscou-se inicialmente identificar por meio do cálculo da média e do desvio padrão as tipologias predominantes nas organizações estudadas conforme dados da Tabela 2 à seguir:

Tabela 2 - Estatísticas descritivas

\begin{tabular}{|l|c|c|c|c|c|}
\hline \multicolumn{1}{|c|}{ Variáveis } & Observações & Média & Desvio-padrão & Mínimo & Máximo \\
\hline Cultura & 240 & 3.063786 & 1.3349 & 1 & 5 \\
\hline Estratégia & 240 & 2.915638 & 1.23318 & 1 & 5 \\
\hline Empregados & 240 & 11.62551 & 6.7349 & 1 & 25 \\
\hline Tempo de Atuação & 240 & 10.69008 & 4.10937 & 2 & 21 \\
\hline
\end{tabular}

Fonte: Dados da pesquisa, 2016. 
Ao observar a Tabela 2, sobre a cultura organizacional presente nas empresas estudadas, identificou-se um valor médio de 3,06, com desvio padrão de 1,3, o que aponta para um perfil cultural predominantemente empreendedor, que segundo Paz e Mendes (2001), representa uma postura de organizações voltadas para inovação, apresentando padrões comportamentais de cultura direcionados à competição em ambientes que estão em fase de constantes mudanças.

No que diz respeito à estratégia organizacional, a tipologia da estratégia apresenta valor médio de 2,92 com desvio padrão de 1,2, o que representa a existência predominante de uma tipologia de estratégia organizacional transitando entre uma postura analítica e prospectiva. Assim, há uma relação direta entre essas características, nas quais contribuem para o tempo de atuação dessas lojas, com média de 10,69 anos, pois nota-se um maior equilíbrio e controle nas decisões estratégicas, além de aliar com aspectos inovadores, a partir da ênfase em investimentos na pesquisa e na criatividade. No entanto, estas características trazem mais riscos para a inovação, pois há um controle firme de custos com flexibilidade e adaptabilidade. Neste aspecto, pode-se correlacionar uma quantidade mediana identificada para o número de funcionários por empresa, 11,62, com um desvio-padrão de 6,73.

A Tabela 3 apresenta os resultados dos 3 modelos de regressão estimados nesta pesquisa. Para o primeiro modelo, foram utilizadas as seguintes variáveis: Cultura Organizacional (CultOrg) sendo mensurada de 1 a 5 , em uma escala que representa o grau de flexibilidade cultural da organização, sendo 5, o nível máximo de flexibilidade, sendo denominada por Paz e Mendes (2008) de cultura empreendedora, e 1, estratégia burocrática; Número de Funcionários (Funcionários); Tempo de atuação da Empresa no mercado (TempoAtuação); além de variáveis dummy representando o porte da empresa, sendo representadas por Pequena Empresa (PequenaEmp); Media Empresa (MediaEmp); Média para Grande (MediaGrandeEmp); e Grande Empresa (GrandeEmp). Também foi incluída a variável Franquia (dummy), sendo atribuído o valor 1 caso a loja em questão seja uma franquia.

Como variável dependente, tem-se a Estratégia adotada pelas organizações, sendo mensurada de 1 a 5, em uma escala que representa o grau de flexibilidade estratégica adotada pela organização, sendo 5 o nível máximo de flexibilidade, denominada por Miles e Snow (2003) de estratégia prospectiva, e 1, estratégia defensiva.

Tabela 3 - Resultados dos modelos de regressão

\begin{tabular}{|c|c|c|c|}
\hline Variáveis & Modelo 1 & Modelo 2 & Modelo 3 \\
\hline Constante & $\begin{array}{c}0.4180937 * \\
(0.252)\end{array}$ & $\begin{array}{c}0.55838 * * * \\
(0.239)\end{array}$ & $\begin{array}{c}0.71467 * * * \\
(0.1686)\end{array}$ \\
\hline CultOrg & $\begin{array}{c}0.6330847 * * * \\
(0.043)\end{array}$ & $\begin{array}{c}0.639781 * * * \\
(0.0423)\end{array}$ & $\begin{array}{c}0.6373834 * * * \\
(0.04208)\end{array}$ \\
\hline Funcionários & 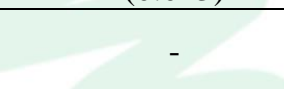 & $\begin{array}{c}0.0323826 * * \\
(0.00846)\end{array}$ & $\begin{array}{c}0.0312 * * * \\
(0.00834)\end{array}$ \\
\hline TempoAtuação & $\begin{array}{c}0.0153723 \\
(0.0136)\end{array}$ & $\begin{array}{c}0.01300 \\
(0.01382)\end{array}$ & - \\
\hline Franquia & $\begin{array}{c}-0.1955414 * * \\
(0.103)\end{array}$ & $\begin{array}{c}-0.1877884 * * \\
(0.09168)\end{array}$ & $\begin{array}{c}-0.18481 * * \\
(0.0912)\end{array}$ \\
\hline PequenaEmp & $\begin{array}{c}0.2071719 \\
(0.177)\end{array}$ & (2) & - \\
\hline MediaEmp & $\begin{array}{c}0.358175^{* *} \\
(0.1810)\end{array}$ & - & - \\
\hline MediaGrandeEmp & $\begin{array}{c}0.1377261 \\
(0.1966)\end{array}$ & - & - \\
\hline GrandeEmp & $\begin{array}{c}0.2071719 * * \\
(0.1773)\end{array}$ & - & - \\
\hline $\mathrm{R}^{2}$ & 0.53 & 0.51 & 0.50 \\
\hline Estatística F & 52.21 & 62.25 & 83.99 \\
\hline Teste de White & $\begin{array}{c}50.35 \\
(0.0351)\end{array}$ & $\begin{array}{c}34.92 \\
(0.0015)\end{array}$ & $\begin{array}{c}30.12 \\
(0.0000)\end{array}$ \\
\hline Shapiro-Wilks & $\begin{array}{l}0.985 \\
(0.15)\end{array}$ & $\begin{array}{c}0.985 \\
(0.015)\end{array}$ & \\
\hline Amostra & 240 & 240 & 240 \\
\hline
\end{tabular}

Fonte: Dados da pesquisa, 2016. 
O modelo 1 apresenta a regressão que levou em consideração todas as variáveis de controle, à exceção do número de funcionários, visto que o porte das empresas é determinado a partir do número de funcionários. Nesta estimação, o coeficiente de explicação $\left(\mathrm{R}^{2}\right)$ é de 0.53 , ou seja, $53 \%$ da variação na estratégia organizacional é explicada pelas variáveis preditoras.

A variável "CultOrg", variável de interesse do estudo, mostrou-se estatisticamente significante a $1 \%$, e seu coeficiente representa que o aumento de uma unidade na escala de cultura organizacional aumenta o grau de flexibilidade estratégica em 0.63 pontos. No que diz respeito ao tempo de atuação da empresa (TempoAtuação), tal variável não se mostrou estatisticamente significante. Em relação às variáveis dummy de porte, apenas médias e grandes empresas apresentaram resultados estatisticamente significantes e positivos, indicando que quanto maior o porte da empresa, maior a sua orientação à uma estratégia empreendedora.

A variável "Franquia" também é estatisticamente significante, entretanto, seu sinal é negativo, indicando que franquias, em média possuem menor grau de flexibilidade estratégica. Foram testadas as hipóteses da normalidade (Shapiro-Wilks), multicolineariedade (Valor inflacionado VIF) e Homoscedasticidade (teste de White). Os resultados indicam que a amostra possui distribuição normal, sem multicolineariedade perfeita, mas apresenta heteroscedasticidade. Desse modo, foi realizada uma estimação robusta para o modelo.

Já o modelo 2 apresenta como variáveis de controle o tempo de atuação, número de funcionários e franquia. $\mathrm{O}$ coeficiente da variável de interesse, cultura organizacional (CultOrg), mostrou-se bastante semelhante ao modelo 1, sendo estatisticamente significante a $1 \%$, e com magnitude de aproximadamente 0.63 pontos. A variável "TempoAtuação" não apresentou significância estatística.

O número de funcionários mostrou-se estatisticamente significante a $5 \%$, com efeito positivo, indicando que empresas de maior porte tendem a ter uma estratégia mais flexível. O coeficiente de explicação do modelo é de 0.51 . Em relação à variável "Franquia", assim como no primeiro modelo, esta variável apresentou efeito negativo em relação ao maior nível de flexibilidade estratégica da empresa, com efeito negativo de 0.18. Este modelo também apresentou heteroscedasticidade.

Por fim, foi estimado o terceiro modelo, utilizando como controles as variáveis "Funcionários" e "Franquia". Os resultados são semelhantes aos modelos apresentados anteriormente, com todas as variáveis estatisticamente significantes, e com coeficientes bastante semelhantes aos modelos anteriores. $\mathrm{O}$ poder de explicação do modelo passou a ser de 0.50. Por apresentar heteroscedasticidade, também foi utilizada uma estimação robusta.

\section{DISCUSSÃO}

Inicialmente, o estudo realizou uma análise descritiva sobre as tipologias de cultura e estratégia organizacional das organizações estudadas. Pelos resultados obtidos, ao considerar que estas organizações possuem um padrão de cultura predominantemente empreendedor, considera-se que elas podem apresentar uma maior flexibilidade a mudanças, pois, a partir desses aspectos culturais elas conseguem se adequar ao seu público-alvo e ao ambiente em que está inserida, consequentemente, pode-se notar o tempo de atuação amadurecido dessas organizações.

No que se refere aos profissionais, encontra-se uma busca constante por reconhecimento e admiração, enfrentando as mudanças como desafios a serem superados, e almejando alcançar seu ideal de perfeição que está contido no ego ideal, sendo essa postura fundamental, visto que o público que frequenta estas organizações é apto a constantes mudanças. Dessa forma, o estilo cultural das lojas é favorável e intrinsecamente relacionável à tipologia de estratégia prospectiva, estando estes resultados alinhados à literatura existente (Johnson, 1992; Tomassini, 2001; Pereira e Nunes, 2002; Degenhardt, 2006; Sampaio et al., 2007).

Em relação ao perfil estratégico, foi observado que as organizações em sua maioria possuem um perfil que se classifica entre o comportamento analítico e o comportamento prospectivo, o que, segundo Miles e Snow (2003), traz uma postura de diferenciação e inovação, equilibrando eficiência e aprendizagem. Estes resultados diferem dos resultados encontrados por Degenhardt (2006), onde a tipologia mais presente foi a estratégia defensiva, característica comum em organizações do segmento industrial.

Outra característica observável dessa transição de tipologias (analisadora para prospectiva) é a eficiência na produção da linha de produtos estáveis. Com estes resultados, fica perceptível que quanto maior a proximidade com a tipologia prospectiva, mais a organização será flexível, descentralizada, estruturalmente adaptável, além de possuir uma forte aptidão à pesquisa (Miles e Snow, 2003).

Ao aplicar o método de regressão múltipla, foi observado que a variável inerente à cultura organizacional se mostrou significante estatisticamente par influenciar no comportamento estratégico das organizações. Este resultado corrobora com outros estudos, quando se analisam as relações entre culturas e estratégias organizacionais, na medida em que culturas organizacionais com um perfil mais centralizador e burocrático tendem a favorecer a adoção de estratégias 
menos flexíveis. No estudo de Pereira e Nunes (2002), por exemplo, foram levantadas percepções de cultura centralizadora encontradas nas organizações estudadas que definem estratégias fragmentadas e pouco compreendidas. Já na pesquisa de Tomassini (2001), na organização estudada, foi verificado que, embora haja uma característica conservadora no modo como a organização conduz a sua estratégia, algumas ações realizadas, oriundas de uma atitude eminentemente proativa dos colaboradores, promovem um direcionamento organizacional para a inovação, apresentando, assim, indícios de influência da cultura sobre a estratégia organizacional.

Outro resultado interessante na pesquisa é que o número de funcionários também contribuiu para a adoção de uma estratégia mais prospectiva das organizações. Este resultado é interessante, quando se compara com o resultado gerado na pesquisa de Reis (2007), na qual pequenas e médias empresas (objeto de estudo da pesquisa) apresentaram a predominância de um modelo estratégico defensivo. Assim, estes estudos confirmam que quanto maior for a organização, maior a possibilidade de adoção de estratégias flexíveis.

\section{CONSIDERAÇÕES FINAIS}

Objetivando mensurar o comportamento estratégico a partir das relações entre os estilos culturais de Paz e Mendes (2008) com as tipologias de estratégias genéricas de Miles e Snow (2003), o presente estudo possibilitou a compreensão e identificação da cultura organizacional, como uma variável independente e a sua influência sobre o comportamento estratégico, variável dependente, em lojas dos shoppings centers da Cidade do Natal/RN.

Desta forma, ao se analisar os estilos culturais presentes nas lojas de shoppings centers, observou-se um predomínio de dois estilos culturais, voltados para um perfil mais inovador, que segundo Paz e Mendes (2008) são os estilos afiliativo e empreendedor, com destaque ao empreendedor. Esses resultados demonstram uma abertura cultural às mudanças naturais do ambiente no qual estão inseridos, favorecendo a criação de políticas de inovação, por conseguinte, as adequações ao seu público-alvo, gerando crescimento que se reflete no tempo de atuação, já amadurecido dessas organizações.

No que tange a mensuração do comportamento estratégico adotado pelas lojas de shopping centers estudadas, notou-se o predomínio de duas estratégias propostas por Miles e Snow (2003), que são as estratégias analisadoras e prospectivas. Traçando um paralelo entre essas duas, pode-se afirmar de que as lojas que apresentaram a tipologia analisadora possuem um perfil mais conservador e controlador com seus processos, que oferece uma maior resistência às mudanças. Em contrapartida, as lojas que apresentaram a tipologia prospectiva se caracterizaram por serem mais flexíveis e adaptáveis às mudanças, focando na área de pesquisa e inovação, sendo favorável ao ambiente de inserção.

Os resultados da análise da regressão múltipla indicaram estatisticamente uma influência do estilo cultural na definição do comportamento estratégico das organizações, resultados estes confirmados ao se observar os percentuais de explicação,do modelo, bem como o valor da estatística $t$ da variável "CultOrg" em relação à variável dependente. Estes resultados confirmam que, considerando os modelos de cultura e estratégia estudados nesta pesquisa, quanto mais flexível for o estilo de cultura organizacional, maior será a capacidade de formação estratégica descentralizada, voltada para a aprendizagem e menos rígida para a mudança no ambiente organizacional, confirmando, portanto, os pressupostos teóricos estabelecidos. Além disto, o estudo também contribuiu no sentido de esclarecer que o tamanho da organização também pode influenciar positivamente na adoção de uma estratégia flexível e de postura inovadora.

Por fim, os resultados identificados com o presente estudo confirmam as suposições da teoria quando apontam uma necessidade de se promover um alinhamento do comportamento estratégico da organização com a sua cultura organizacional, sendo esta cultura uma das questões relevantes para a implantação de uma estratégia organizacional eficaz.

Contudo, é importante considerar que esta pesquisa apresenta algumas limitações. Em primeiro lugar, considera-se que, pelo fato de a pesquisa englobar a realidade de lojas de shopping centers, algumas características evidenciadas podem não ser tão presentes em outras realidades, pela natureza do negócio estudado. Por exemplo, o contato direto com o cliente final pode ser uma questão importante no sentido de promover um melhor entendimento sobre o mercado e a necessidade de mudança constante em termos de direcionamento e comportamento estratégico. Além disso, a própria cultura inerente a este perfil organizacional pode assumir características inerentes a este cenário. Deste modo, sugere-se estudos que abordem esta temática em outras realidades, como, por exemplo, em setores mais tradicionais, afim de se poder verificar se estes resultados de fato se repetem ou se são característicos deste presente objeto de estudo. Além disso, cabe também estudar estes fatores externos como condicionantes de uma postura e formação cultural organizacional destas corporações.

Outra limitação importante se refere à forma como os dados desta pesquisa foram analisados. Considerando que os perfis de cultura organizacional e de comportamento estratégico foram definidos em faixas de valores obtidos, não houve uma captação real de como os diferentes tipos de cultura e de estratégia podem estar vinculados com outras variáveis de controle, de modo que se possa entender melhor como determinados grupos de organizações com determinado perfil cultural, por exemplo, podem estar associados a outras características. Outros estudos podem esclarecer melhor esta relação. 
Por fim, considera-se que este estudo foi limitado no sentido de avaliar as relações entre a cultura das organizações estudadas e o seu comportamento estratégico a partir de um estudo transversal. Considerando que o mercado global e a conjuntura ambiental das corporações são dinâmicas, é importante observar que determinadas questões podem influenciar a forma como as empresas se estruturam, definem sua identidade e sua forma de atuação ao longo do tempo. Deste modo, sugere-se que outros estudos investiguem esta relação de maneira longitudinal, abrangendo aspectos que poderão interferir na influência da cultura sobre o padrão de comportamento estratégico.

\section{REFERÊNCIAS}

Abrasce - Associação brasileira de shopping centers (2014). Manual de estratégia. Disponível em: <http://www.portaldoshopping.com.br/>. Acesso em: 15 de julho de 2014 .

Afonsêca, S. M., Teodósio, A. D., \& Paixão, B. O. (2011). Paradoxos e dilemas na construção de estratégias: possibilidades interpretativas a partir da noção de papéis nas organizações. Revista Gestão \& Tecnologia, 11 (2): 1-14.

Almeida, L. F., Irber, F. M. V. F., \& Souza, L. J. S. S. (2013). O papel da cultura organizacional: Métodos de avaliação da influência da cultura de inovação nas organizações. Artigo apresentado no XXXIII Encontro Nacional de Engenharia de Produção (ENEGEP). Salvador (BA), Brasil.

Bott, M. G., Silva, P. A., \& Sassaki, A. H (2006). Uma abordagem estratégica baseada na tipologia de Miles e Snow. Artigo apresentado no II Etic Encontro de Iniciação Científica. Presidente Prudente, Brasil.

Cameron, K. S. \& Quinn, R. E. (2006). Diagnosing and changing organizational culture. San Francisco: Jossey-Bass.

Carvalho, C. E. \& Ronchi, C. C. (2005). Cultura organizacional: teoria e pesquisa. Rio de Janeiro: Fundo de Cultura.

Castro, J. M., \& Silva, C. L. O. (2012). A Influência da Cultura Organizacional e da Orientação Estratégica na Gestão de Recursos Humanos: um estudo de caso. Revista de Administração da Unimep-Unimep Business Journal, 10(1), 1-28.

Collis, J. \& Hussey, R. (2005). Pesquisa em Administração: um guia prático para alunos de graduação e pós-graduação, 2. ed. Porto Alegre: Bookman.
Conant, J. S., Mokwa, M. P., \& Varadarajan, P. R. (1990). Strategic types, distinctive marketing competencies and organizational performance: a multiple measures-based study. Strategic Management Journal, 11 (5): 365-383.

Creswell, J. W. (2010). Projeto de pesquisa: métodos qualitativo, quantitativo e misto, 3. ed.. Porto Alegre: Artmed.

Daft, R. L. Organizações: teorias e projetos (2002). São Paulo: Thomson.

Degenhardt, V. W (2006). Estratégia competitiva e cultura organizacional: um estudo exploratório do estágio atual da indústria brasileira de equipamentos mecânicos pesados. Dissertação de mestrado em Administração. Pontifícia Universidade Católica de São Paulo, São Paulo.

Dutra, J. S., Fleury, M. T. L., \& Ruas, R (2006). Competências: Conceito, Métodos e Experiências. São Paulo: Atlas.

Ferreira, E. R. A., Bubach, C., \& Silva Júnior, A. (2014). A estratégia organizacional como uma prática social: análise bibliométrica no período de 2003 a 2013. Artigo publicado no Encontro de Estudos Organizacionais da ANPAD (ENEO), Gramado-RS, Brasil.

Fleury, M. T. L. \& Fischer, R. M. (ed.) (2007). Cultura e poder nas organizações, 2. ed. São Paulo: Atlas, 15-27.

Fonseca, J. S., \& Martins, P. A. (1996). Curso de Estatística, 6. ed. São Paulo: Atlas.

Gaeta, A. C (1992). Gerenciamento dos shoppings centers e transformação do espaço urbano. In Pintaudi, S. M. \& Frúgoli Jr., H. (ed). Shopping centers: espaço, cultura e modernidade nas cidades brasileiras. São Paulo: Unesp.

Hair Jr., J. F. et al (2005). Análise multivariada de dados, 5. ed. Porto Alegre: Bookman.

Hambrick, D. C. (1980). Operationalizing the concept of business-level strategy in research. Academy of Management Review, 5 (4): 567-575.

Heinzmann, L. M., \& Machado, D. D. P. N. (2015). Cultura Organizacional e estágios de internacionalização: um estudo em quatro empresas do segmento de metal-mecânico-elétrico brasileiro. Brazilian Business Review, 11 (2): 35-66. 
Johnson, G. (1992). Managing strategic changestrategy, culture and action. Long range planning, 25(1), 28-36.

Klein, A. (2008). Organizational culture as source of competitive advantage. Bangkok: E-Leader.

Kotler, P., \& Armstrong, G. (1994). Principles of Marketing. New Jersey: Prentice Hall.

Lang, J. et al. (2014). Percepção do ambiente organizacional e comportamento estratégico dos gestores de IES. Revista Brasileira de Estratégia REBRAE, 6 (1): 56-73.

Lourenço, C. D. S., \& Ferreira, P. A. (2012). Cultura organizacional e mito fundador: Um estudo de caso em uma empresa familiar. Gestão e Regionalidade, 28 (84): 61-76.

Martins, T. S. et al.(2008). A Influência da Tipologia Estratégica de Miles e Snow no Grau de Orientação para o Mercado em Instituições de Ensino Fundamental e Médio do Estado do Paraná. Revista Ibero-Americana de Estratégia, 7 (2), 125-137.

Martins, T. S. et al. (2014). Um quadro analítico para a tipologia e capacidades dinâmicas de Miles e Snow. Revista Ibero-Americana de Estratégia, 13 (1): 2233.

Miles, R. E., Snow, C. C. (2003). Organizational strategy, structure, and process. Stanford. Stanford Business Books.

Mintzberg, H., Ahlstrand, B., \& Lampel, J. (2000). Safári da estratégia: um roteiro pela selva do planejamento estratégico. Porto Alegre: Bookman.

Mintzberg, H. et al. (2006). O processo da estratégia: conceitos, contextos e casos selecionados, 4. ed. Porto Alegre: Bookman.

Monteiro, A. S., Palma, P. J., \& Lopes, M. P. (2012). Como os gigantes aprendem a dançar: o papel mediador do capital empreendedor na relação entre cultura de inovação e desempenho. Revista de Administração e Inovação, 9 (4): 44-67.

Motta, F. C. P. \& Caldas, M. P. (ed.) (1997). Cultura Organizacional e Cultura Brasileira. São Paulo: Atlas.

Paz, M. G. T \& Mendes, A. M. B. (2008). Estilos de funcionamento organizacional. In Siqueira, M. M. M. (ed.) Medidas do comportamento organizacional: ferramenta de diagnóstico e de gestão. Porto Alegre: Artmed.
Paz, M. G. T., Mendes, A. M. B., \& Gabriel, I. (2001). Configurações de poder organizacional e estilos de caráter. Psicologia: organizações e trabalhos, 1 (1): 141-186.

Pedrozo, E. Z. \& Rodriguez, I. A. C. (2013). Aproximações entre pensamento complexo e estratégia organizacional. Teoria e Prática em Administração, 3 (1): 1-8.

Pereira, J. A. G. \& Nunes, R. S. (2002). Cultura organizacional e estratégia competitiva: um estudo de multicasos nas concessionárias de automóveis de Santa Maria (RS). Administração on-line - FECAP, 3 (4): 21.

Pettigrew, A. M. A cultura das organizações é administrável? (2007) In Fleury, M. T. L. \& Fischer, R. M. (ed.) Cultura e poder nas organizações, 2. ed. São Paulo: Atlas.

Porter, M. E. (1986). Estratégia competitiva. Rio de Janeiro: Campus.

Powell, T. C. Strategic management and the person (2014). Strategic Organization, 12 (3): 200-207.

Ravishankar, M. N., Pan, S. L., \& Leidner, D. E. (2011). Examining the strategic alignment and implementation success of a KMS: A subculturebased multilevel analysis. Information Systems Research, 22(1), 39-59.

Reis, J. A. F. (2007). Tipos de estratégias e estilos cognitivos gerenciais: um estudo dde suas comparações em indústrias de pequeno e médio porte. Dissertação de mestrado em Administração. Pontífica Universidade Católica do Paraná, Curitiba, 2007.

Robbins, S. P. (2005). Comportamento Organizacional, 11. ed. São Paulo: Pearson Prentice Hall.

Sampaio, D. O., Freitas, A. F., \& Máximo, M. S. (2007). O relacionamento entre estratégia e cultura organizacional em cooperativas e associações da cidade de Viçosa em Minas Gerais. Artigo apresentado no XLV Congresso da Sociedade Brasileira de Economia, Administração e Sociologia Rural, Londrina (PR), Brasil.

Schein, E. H. (2009). Cultura Organizacional $e$ liderança. São Paulo: Atlas.

Scherer, F. L. \& Mussi, C. W. (2000). Comportamento Estratégico: Um Estudo na Indústria de Plásticos de Santa Catarina. Revista de Ciências da Administração, (2) 4: 65-75. 
Srour, R. H. (1998). Poder, cultura e ética nas organizações. Rio de Janeiro: Campus.

Tomassini, N. L. S. (2001). A influência da cultura organizacional na administração estratégica de uma empresa estatal: um estudo de caso. Dissertação de mestrado em Engenharia da Produção. Universidade Federal de Santa Catarina, Florianópolis.

Vidal, D. M., Barbosa, J. G. P., \& Bouzada, M. A. C. (2014). A relação entre pressão ambiental e comportamento estratégico: uma pesquisa em MPE do setor de autopeças. Revista Ibero-Americana de Estratégia, 13 (3): 48-62.
Wooldridge, J. M. (2013). Introdução à econometria: uma abordagem moderna, 4. ed. São Paulo: Cengage Learning.

Zaccarelli, S. B. (1986). Programação e controle da produção. São Paulo: Pioneira.

Zago, C. C. Cultura organizacional: formação, conceito e constituição (2013). Sistemas \& Gestão. 8, (2): 106-117.

Zanin, E. R. M., Machado, N. S., \& Sehnem, S. (2011). Comportamento estratégico segundo a teoria de Miles e Snow: estudo de caso em agroindústria do oeste de Santa Catarina. Revista de Gestão Organizacional, 4 (2): 87-101. 